\title{
Sensitivity Profile of Carbapenem-Resistant Enterobacteriaceae and Therapeutic Outcome in Children in Syria
}

\author{
Rama Jadeed ${ }^{1, *}$ and Isam Anjak ${ }^{2}$ \\ ${ }^{1}$ M.D. Faculty of Medicine, Damascus University, Syria \\ ${ }^{2}$ Professor of Pediatrics Infectious Disease, Faculty of Medicine, Damascus University, Syria \\ *Corresponding Author. Email: jadeedrama@gmail.com
}

\begin{abstract}
Carbapenem-Resistant Enterobacteriaceae (CRE) threaten public health because the infections they cause carry a high mortality rate and limited treatment options. Few studies focus on these organisms in children compared to the number of the ones carried out on adults. Therefore, the importance of our research is to contribute to broadening our knowledge on the effects of these organisms in children. The objective of our research was to determine each of the following: the sensitivity profile of CRE, available treatment options for dealing with these bacteria, the therapeutic outcome, and the factors affecting this outcome. Our study design was a prospective, single-centre analytical cohort study. Samples were collected from the Children's University Hospital in Damascus, which conatins 389 beds and 60 incubators. Disk diffusion was used to examine bacterial susceptibility; some samples were tested in BD Phoenix system. Our research examined antibiotics resistance rates, duration to treatment response, and mortality rate. The research sample included 277 samples, 125 of which were resistant to Carbapenem. High resistance to most of the available antibiotics characterized the Carbapenem Resistant Enterobacteriaceae strains. Colistin, Imipenem and Amikacin were the most used antibiotics in our study; one or two antibiotics were used in most cases. Moreover, the mortality rate was $42.4 \%$, and most of the deaths were due to infection itself. The median duration of treatment response was 8.4 days, and the factors were associated with a statistically significant higher mortality rate (age less than one month, the use of certain antibiotics in treatment such as Piperacillin-Tazobactam, and association with respiratory tract infection). Regarding response to treatment, there was no significant difference between Resistant and Non-Resistant Carbapenem Enterobacteriaceae in terms of duration. Enterobacteriaceae resistance to Carbapenems caused a very high mortality rate. Our treatment options were limited; consequently, the introduction of novel antibiotics in our treatment options is required.
\end{abstract}

Keywords: Carbapenem-Resistant Enterobacteriaceae (CRE), Outcome, Children.

\section{INTRODUCTION}

Carbapenem Resistant Enterobacteriaceae (CRE) are opportunistic bacteria that cause severe infections acquired in hospitals, and the spread of Carbapenemase Producing Enterobacteriaceae (CPE) has threatened public health worldwide. For example, Klebsiella Pneumoniae Resistant to Meropenem and Imipenem increased rapidly from $2.9 \%$ and $3 \%$ in 2015 to $26.3 \%$ and $25 \%$ in 2018, respectively. In Europe, Klebsiella Pneumoniae, which is resistant to Carbapenems, is most common in the Balkan and Mediterranean countries; the rates were $40 \%$ in Italy, and $60 \%$ in Greece. The majority of recent studies target CRE in adults, whereas only a small number is available to examine the prevalence of these strains in children [1].
The spread of resistance to Carbapenems is a matter of concern in Italy, where one third of the Klebsiella Pneumoniae strains, which are isolated from blood and CSF, are resistant to Carbapenems [2].

In 2015, the Centers for Disease Control and Prevention (CDC) determined the definition of Carbapenem Resistant Enterobacteriaceae as either being resistant to at least one of the Carbapenems (Imipenem, Meropenem, Doripenem or Ertapenem) based on sensitivity tests without indicating their resistance to Cephalosporins, or being proven to produce one of the Carbapenemase enzymes [3]. CRE can infect many sites in the body, such as the urinary tract, lungs, abdomen, surgical sites, and the bloodstream. The genes that cause resistance to Carbapenem do not lead to an increase in 
morbidity, so there are no differences in initial manifestations between resistant and less resistant species of this pathogen. The main indicator of infections caused by these organisms might be a result of a lack of disease control. This leads to a more progressive condition despite initial antibiotics coverage [4].

The mortality rate caused by CRE is as high as $40 \%$ or more in adults, which approximates the mortality rates of the pre-antibiotic period [5]. On the other hand, children mortality rate was lower at $10 \%$ [6].

The presence or absence of Carbapenemase production ought to not direct the choice of treatment. In general, the plan of antibiotics selection should be placed for Carbapenem Resistant Enterobacteriaceae that produce and that do not produce Carbapenemase. It is possible that the optimal treatment strategy will vary according to the production or absence of Carbapenemase, but current information is not sufficient to establish this method of directing treatment [7].

Current treatment choices include:

Colistin: it is active against most of these bacteria. This activity is due to its high positive charge. Bactericidal depends on concentration, is rapid and occurs 5 minutes after exposure to Colistin [8].

Tigecycline: it is a bacteriostatic, anti-bacterial drug. It can be used in the treatment of Carbapenem Resistant Enterobacteriaceae that produce Metallo-BetaLactamase enzyme. The recommended drug dosage is 2$3 \mathrm{mg} / \mathrm{kg} / \mathrm{a}$ dose every 12 hours. It is suggested to use it as synergic treatment in blood stream infections and very ill children. We use the single treatment for mild infections, and it is not recommended in urinary tract infections if there is sensitivity to other antibiotics [9] [7].

Aminoglycosides: When effective in vitro, aminoglycosides are an available option for the single or combination treatment of CRE infections. In spite of the limitations imposed by toxic effects, several studies described the effectiveness of aminoglycosides in vitro, where the sensitivity to Gentamicin ranged between 35$62.7 \%$, to Amikacin between $16-82.3 \%$ and to Tobramycin between $61-98 \%$. This variation may depend on the CRE strains [10].

Iravacycline: it has efficacy in vitro against multidrug-resistant organisms since it is twice as effective as Tigecycline against CRE in adults [11]. It is active against CRE infections except urinary tract infections and septicemia; in children, a study is underway under the code (NCT03696550) [7].

Fosfomycin: it is effective against most strains of Carbapenem Resistant Enterobacteriaceae. Its intravenous form has been used to treat the infection of these bacteria with success in adults outside the United States of America, and the intravenous dose is available for children [12] [13].
Novel antibiotics: they include CeftazidimeAvibactam, Meropenem-Vaborbactam, and ImipenemRelebactam [7].

\section{MATERIAL AND METHODS}

This study was conducted at the Children's University Hospital in Damascus, and it is a prospective cohort analytical study. The samples were collected between the dates of 1/6/2019 and 1/6/2020. They were from blood, urine, cerebrospinal fluid, swab of pus, bronchial lavage, peritoneal fluid, catheter, and cerebral shunt. We have used these culture media (blood agar, Eosin Methylene Blue, chocolate agar, thioglycolate, BD BACTEC bottle) to determine bacterial species. Disk diffusion was used to examine bacterial susceptibility; the results were interpreted according to BD charts [14]. Some samples were tested in BD Phoenix system. We excluded samples of urine when the number of bacterial colony count was less than 105CFU $\backslash \mathrm{ml}$ because almost all urine samples were taken from urine bags. Samples were taken from all the following sections of the hospital: intensive care unit of neonate, the general ward (in which there are neurologic, digestive, cardiac, endocrinology, musculoskeletal, metabolic and renal diseases), infectious disease ward, intensive care unit beyond neonate, neonatal ward, tumors and blood diseases ward, and surgery ward. We excluded samples that were taken from the emergency department without the admission of the patient. The data were collected after taking informed consent. These data included demographic information (age, gender), bacterial species, place of admission, the type of antibiotic used in the treatment and the number of antibiotics used. They also consisted of the response to treatment (it was detected as laboratory response by obtaining a sterile culture or clinical response in the event of inability to obtain a new sample as in the case of bronchial lavage), and deaths due to either infection or other causes.

Statistical analysis: the obtained sample size was estimated to be 277 according to Steven Sampson's equation. The data were collected using EXCEL 2010 program. The statistical program SPSS version 26 was used for data processing and statistical analysis. For continuous variables (mean, standard deviation, and the minimum and maximum value were calculated), chi square test and Fisher Exact test were used to compare categorical variables with a significance value of $\mathrm{P}<$ 0.05 .

\section{RESULTS}

The research included 277 samples, 125 (45.1\%) of which were resistant to Carbapenem. The median age was 18 (35) months. Klebsiella was the most prevalent sample. It was 64 (51.2\%), and then E.coli was 54 
(43.2\%). The rest of the samples were Serratia 4 (3.2\%) and Enterobacter 3 (2.4\%).

Table 1 shows the antibiotic susceptibilities of Carbapenem-Resistant Enterobacteriaceae and NonCarbapenem-Resistant Enterobacteriaceae. In the CRE group, the highest resistance rates were to Cephalosporins $(92.8 \%-99.1 \%)$ and Penicillins $(96.8 \%$ $-99.2 \%$ ), while the lowest resistance rates were to Tigecycline $13.3 \%$ and Colistin $26.6 \%$. On the other hand, in the Non-CRE group, the highest resistance rates were to Cefotaxime $82.2 \%$ and Ceftriaxone $82.9 \%$, whereas the lowest resistance rates were to Carbapenems $0 \%$, to Colistin $7.2 \%$ and to Amikacin $7.2 \%$.
(18.4\%), $19(12.5 \%)$ of which were due to infection itself. Table 3 shows the relationship between mortality and the following risk factors: gender, age, sample type, bacterial species, antibiotics number, and antibiotic type. We found that the statistically significant higher risk of fatal outcome age was less than one month $(\mathrm{P}=.003)$, Bronchial lavage was $(\mathrm{P}=.002)$, no antibiotics use was $(\mathrm{P}=.030)$, the use of Piperacillin-Tazobactam in treatment was $(\mathrm{P}=.001)$. The presence of Amikacin in the treatment was associated with statistical significance of a lower mortality rate $(\mathrm{P}=.004)$.

The median duration of the response was 8.4 (5.5) days. The survival curve shown in Fig. 1. reveals no

Table 1. Antibiotic Susceptibilities of Carbapenem-Resistant Enterobacteriaceae and non-Carbapenem-Resistant Enterobacteriaceae

\begin{tabular}{|c|c|c|c|c|c|c|c|}
\hline \multirow{2}{*}{\multicolumn{2}{|c|}{$\begin{array}{l}\text { Antibiotic Susceptibilities of } \\
\text { Carbapenem-Resistant } \\
\text { Enterobacteriaceae and non- } \\
\text { Carbapenem-Resistant } \\
\text { Enterobacteriaceae }\end{array}$}} & \multicolumn{3}{|c|}{ CRE } & \multicolumn{3}{|c|}{ Non-CRE } \\
\hline & & \multirow{2}{*}{$\begin{array}{l}\text { Resistance } \\
\text { No. }(\%) \\
86(68.8 \%)\end{array}$} & \multirow{2}{*}{$\begin{array}{c}\text { Intermediate } \\
\text { No. }(\%)\end{array}$} & \multirow{2}{*}{$\begin{array}{c}\begin{array}{c}\text { Susceptible } \\
\text { No. }(\%)\end{array} \\
38(30.4 \%)\end{array}$} & \multirow{2}{*}{$\begin{array}{l}\text { Resistance } \\
\text { No. }(\%) \\
11(7.2 \%)\end{array}$} & \multirow{2}{*}{$\begin{array}{l}\text { Intermediate } \\
\text { No. }(\%)\end{array}$} & \multirow{2}{*}{$\begin{array}{l}\text { Susceptible } \\
\text { No. }(\%) \\
138 \\
(90.8 \%) \\
\end{array}$} \\
\hline Aminoglycosides & Amikacin & & & & & & \\
\hline & Gentamicin & $113(90.4 \%)$ & $0(0 \%)$ & $12(9.6 \%)$ & $72(47.4 \%)$ & $0(0 \%)$ & $80(52.6 \%)$ \\
\hline \multirow[t]{4}{*}{ Cephalosporins } & Cefotaxime & $\begin{array}{l}114 \\
(99.1 \%) \\
\end{array}$ & $1(0.9 \%)$ & $0(0 \%)$ & $\begin{array}{l}126 \\
(82.9 \%) \\
\end{array}$ & $5(3.3 \%)$ & $21(13.8 \%)$ \\
\hline & Ceftriaxone & $\begin{array}{l}123 \\
(98.4 \%) \\
\end{array}$ & $1(0.8 \%)$ & $1(0.8 \%)$ & $\begin{array}{l}125 \\
(82.2 \%) \\
\end{array}$ & $6(4 \%)$ & $21(13.8 \%)$ \\
\hline & Ceftazidime & $\begin{array}{l}121 \\
(96.8 \%) \\
\end{array}$ & $0(0 \%)$ & $4(3.2 \%)$ & $87(57.2 \%)$ & $17(11.2 \%)$ & $48(31.6 \%)$ \\
\hline & Cefepime & $\begin{array}{l}116 \\
(92.8 \%)\end{array}$ & $3(2.4 \%)$ & $6(4.8 \%)$ & $73(48 \%)$ & $23(15.1 \%)$ & $56(36.9 \%)$ \\
\hline \multirow[t]{2}{*}{ Penicillins } & $\begin{array}{l}\text { Amoxicillin- } \\
\text { Clavolanate }\end{array}$ & $\begin{array}{l}124 \\
(99.2 \%)\end{array}$ & $0(0 \%)$ & $1(0.8 \%)$ & $\begin{array}{l}105 \\
(69.1 \%) \\
\end{array}$ & $17(11.2 \%)$ & $30(19.7 \%)$ \\
\hline & $\begin{array}{l}\text { Piperacillin- } \\
\text { Tazobactam }\end{array}$ & $\begin{array}{l}121 \\
(96.8 \%)\end{array}$ & $0(0 \%)$ & $4(3.2 \%)$ & $35(23 \%)$ & $17(11.2 \%)$ & $\begin{array}{l}100 \\
(65.8 \%)\end{array}$ \\
\hline \multirow[t]{3}{*}{ Quinolones } & Levofloxacin & $99(79.8 \%)$ & $2(1.6 \%)$ & $23(18.6 \%)$ & $51(33.5 \%)$ & $0(0 \%)$ & $\begin{array}{l}101 \\
(66.5 \%)\end{array}$ \\
\hline & Ciprofloxacin & $\begin{array}{l}111 \\
(88.8 \%)\end{array}$ & $2(1.6 \%)$ & $12(9.6 \%)$ & $62(40.8 \%)$ & $21(13.8 \%)$ & $69(45.4 \%)$ \\
\hline & Norfloxacin & $39(95.1 \%)$ & $0(0 \%)$ & $2(4.9 \%)$ & $22(24.2 \%)$ & $36(39.5 \%)$ & $33(31.3 \%)$ \\
\hline \multirow[t]{4}{*}{ Carbapenems } & Imipenem & $58(46.4 \%)$ & $0(0 \%)$ & $67(53.6 \%)$ & $0(0 \%)$ & $0(0 \%)$ & $152(100 \%)$ \\
\hline & Meropenem & $66(528 \%)$ & $2(1.6 \%)$ & $57(45.6 \%)$ & $0(0 \%)$ & $1(0.7 \%)$ & $\begin{array}{l}151 \\
(99.3 \%) \\
\end{array}$ \\
\hline & Ertapenem & $\begin{array}{l}116 \\
(92.8 \%) \\
\end{array}$ & $6(4.8 \%)$ & $3(2.4 \%)$ & $0(0 \%)$ & $7(4.6 \%)$ & $\begin{array}{l}145 \\
(95.4 \%) \\
\end{array}$ \\
\hline & Doripenem & $120(96 \%)$ & $0(0 \%)$ & $5(4 \%)$ & $0(0 \%)$ & $6(4 \%)$ & $146(96 \%)$ \\
\hline \multicolumn{2}{|c|}{ Trimethoprime-sulfamethoxazole } & $115(92 \%)$ & $0(0 \%)$ & $1(08 \%)$ & $57(40.7 \%)$ & $6(4.3 \%)$ & $77(55 \%)$ \\
\hline \multicolumn{2}{|l|}{ Nitrofurantoin } & $24(52.2 \%)$ & $2(4.4 \%)$ & $20(43.4 \%)$ & $9(9.7 \%)$ & $45(48.3 \%)$ & $39(42 \%)$ \\
\hline Polymyxins & Colistin & $33(26.6 \%)$ & $2(1.6 \%)$ & $89(71.8 \%)$ & $11(7.2 \%)$ & $2(1.3 \%)$ & $\begin{array}{l}139 \\
(91.5 \%) \\
\end{array}$ \\
\hline \multicolumn{2}{|l|}{ Fosfomycin } & $32(59.3 \%)$ & $0(0 \%)$ & $22(40.7 \%)$ & - & - & - \\
\hline \multicolumn{2}{|l|}{ Tigecycline } & $2(13.3 \%)$ & $6(40 \%)$ & $7(46.7 \%)$ & - & - & - \\
\hline
\end{tabular}

Colistin was the antibiotic that was used the most in CRE treatment 49 (39.2\%). It was used in combination by $65.3 \%$. Table 2 shows antibiotics used in CRE treatment.

The mortality rate in the CRE group was $53(42.4 \%)$, $38(71.7 \%)$ of which were due to infection itself. On the other hand, the ratio in the Non-CRE group was 28 
Table 2. Antibiotics used in CRE treatment.

\begin{tabular}{|c|c|c|c|c|c|c|}
\hline \multirow{2}{*}{\multicolumn{2}{|c|}{$\begin{array}{l}\text { Antibiotics Used } \\
\text { In Cre Treatment. }\end{array}$}} & \multicolumn{2}{|l|}{ Yes } & \multicolumn{3}{|c|}{ Combination } \\
\hline & & \multirow{2}{*}{$\because$ 름 } & \multirow{2}{*}{ 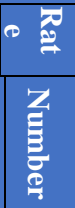 } & \multirow{2}{*}{ 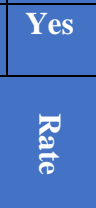 } & \multicolumn{2}{|c|}{ No } \\
\hline & & & & & 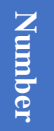 & $\begin{array}{l}\pi \\
\tilde{0}\end{array}$ \\
\hline Colistin & 50 & $40 \%$ & 33 & $66 \%$ & 17 & $34 \%$ \\
\hline Imipenem & 35 & $28 \%$ & 21 & $60 \%$ & 14 & $40 \%$ \\
\hline Meropenem & 29 & $\begin{array}{l}23.2 \\
\% \\
\end{array}$ & 18 & $\begin{array}{l}62.1 \\
\% \\
\end{array}$ & 11 & $\begin{array}{l}37.9 \\
\% \\
\end{array}$ \\
\hline Ciprofloxacin & 8 & $6.4 \%$ & 8 & $100 \%$ & 0 & $0 \%$ \\
\hline Levofloxacin & 4 & $3.2 \%$ & 3 & $75 \%$ & 1 & $25 \%$ \\
\hline Amikacin & 36 & $\begin{array}{l}28.8 \\
\% \\
\end{array}$ & 24 & $\begin{array}{l}66.7 \\
\% \\
\end{array}$ & 12 & $\begin{array}{l}33.3 \\
\% \\
\end{array}$ \\
\hline Gentamicin & 5 & $4.0 \%$ & 2 & $40 \%$ & 3 & $60 \%$ \\
\hline Cefotaxime & 2 & $1.6 \%$ & 2 & $100 \%$ & 0 & $0 \%$ \\
\hline Ceftriaxone & 2 & $1.6 \%$ & 0 & $0 \%$ & 2 & $\begin{array}{l}100 \\
\%\end{array}$ \\
\hline Ceftazidime & 3 & $2.4 \%$ & 3 & $100 \%$ & 0 & $0 \%$ \\
\hline Cefepime & 6 & $4.8 \%$ & 5 & $\begin{array}{l}83.3 \\
\% \\
\end{array}$ & 1 & $\begin{array}{l}16.7 \\
\%\end{array}$ \\
\hline $\begin{array}{l}\text { Piperacillin- } \\
\text { Tazobactam }\end{array}$ & 10 & $8.0 \%$ & 10 & $100 \%$ & 0 & $0 \%$ \\
\hline $\begin{array}{l}\text { Trimethoprime- } \\
\text { Sulfamethoxaz } \\
\text { ole }\end{array}$ & 2 & $1.6 \%$ & 1 & $50 \%$ & 1 & $50 \%$ \\
\hline
\end{tabular}

\section{DISCUSSION}

During the past decade, there has been a frightening global increase in the incidence and prevalence of Carbapenem Resistant Enterobacteriaceae. Only 37\% of the Member States could provide WHO with data; there was some variation in the recorded geographical distribution of the Carbapenem Resistant Enterobacteriaceae [15]. According to our knowledge, this is the first research on CRE in Syria conducted in pediatrics.

The rate of CRE was higher than other studies in which the rate was about $21 \%$ [16]. The majority of CRE species were Klebsiella then E.coli. These ratios are close to the ones in Saudi Arabia [17]. Moreover, Klebsiella was the most common CRE species with a lower ratio for E.coli in Italy, which was similar to our study [18]. Enterobacter was the most common CRE species in research on children in the USA, and Klebsiella was in the second place with less ratios for E.coli [6] [19] [20].

According to antibiotic sensitivity, CRE showed high resistance ratios to almost all our available antibiotics. The resistance rates to Aminoglycosides were (Amikacin $68.8 \%$ and Gentamycin 90.4\%). These ratios were partially similar to the Logan study conducted in the USA in 2012; the resistance rates to Aminoglycosides were $66 \%$ [6]. However, the resistance rates in our research are clearly higher than the resistance rates in the Montagnani study in Italy; Amikacin and Gentamicin resistance rates were $27.5 \%$ and $44.1 \%$, respectively [18]. Furthermore, in general, the resistance rate to
Aminoglycosides in the Logan 2015 study in the USA was $52.26 \%$ [19]. The resistance to Amikacin was $49.6 \%$ and to Gentamicin was $67.9 \%$ [1] in the Han study conducted in China. Nevertheless, the resistance rates were low in the Chiotos study in the U.S.A. They were 5\% for Amikacin and 30\% for Gentamicin [20].

Likewise, the resistance rates were very high for Cephalosporins (Cefotaxime 99.1\%, Ceftriaxone 98.4\%, Ceftazidime $96.8 \%$ and Cefepime $92.8 \%$ ). These ratios are close to the Montagnani study [18], and the Han study [1], while they were less than the resistance rates for Cefepime in the Logan 2015 study where they were $51.8 \%$ [19]. In addition, the resistance rates to Penicillins with Beta-Lactamase were high in our study $(99.2 \%$ to Amoxicillin with Clavulanate and $96.8 \%$ to Piperacillin with Tazobactam). This is consistent with the Logan 2015 study [19], the Logan 2012 study [6], and the Han study [1].

Table 3. Relationship between mortality and risk factors.

\begin{tabular}{|c|c|c|c|}
\hline \multicolumn{2}{|c|}{$\begin{array}{l}\text { Relationship between mortality and } \\
\text { those risk factors }\end{array}$} & \multirow{2}{*}{$\begin{array}{l}\text { Mortality } \\
\text { no.(rate) } \\
33(47.8 \%)\end{array}$} & \multirow{3}{*}{$\begin{array}{l}\text { P- } \\
\text { value } \\
0.173\end{array}$} \\
\hline \multirow[t]{2}{*}{ gender } & Male $(n=69)$ & & \\
\hline & Female $(n=56)$ & $20(35.7 \%)$ & \\
\hline \multirow[t]{5}{*}{ age } & $0-1 \mathrm{~m}(\mathrm{n}=56)$ & $32(57.1 \%)$ & 0.003 \\
\hline & $1-12 m(n=40)$ & $9(22.5 \%)$ & 0.002 \\
\hline & $1-3 y(n=8)$ & $2(25 \%)$ & 0.465 \\
\hline & $3-6 y(n=8)$ & $4(50 \%)$ & 0.721 \\
\hline & $6-13 y(n=13)$ & $6(46.1 \%)$ & 0.772 \\
\hline \multirow[t]{6}{*}{ Sample type } & Blood $(n=46)$ & $22(47.8 \%)$ & 0.349 \\
\hline & Urine $(n=31)$ & $5(16.1 \%)$ & 0.001 \\
\hline & Bronchial lavage $(n=26)$ & $18(69.2 \%)$ & 0.002 \\
\hline & Pus $(n=14)$ & $5(35.7 \%)$ & 0.591 \\
\hline & cerebrospinal fluid $(\mathrm{n}=7)$ & $2(28.6 \%)$ & 0.698 \\
\hline & Peritoneal dialysis $(n=1)$ & $1(100 \%)$ & 0.424 \\
\hline \multirow{4}{*}{$\begin{array}{l}\text { Bacterial } \\
\text { specie }\end{array}$} & Klebsiella $(n=64)$ & $27(42.2 \%)$ & 0.961 \\
\hline & E. coli $(n=54)$ & $24(44.4 \%)$ & 0.687 \\
\hline & Serratia $(n=4)$ & $2(50 \%)$ & 1 \\
\hline & Enterobacter $(n=3)$ & $0(0 \%)$ & 0.261 \\
\hline \multirow{5}{*}{$\begin{array}{l}\text { Antibiotics } \\
\text { number }\end{array}$} & $0(n=4)$ & $4(100 \%)$ & 0.030 \\
\hline & $1(n=63)$ & $19(30.2 \%)$ & 0.005 \\
\hline & $2(n=48)$ & $25(52.1 \%)$ & 0.084 \\
\hline & $3(n=8)$ & $4(50 \%)$ & 0.721 \\
\hline & $4(n=2)$ & $1(50 \%)$ & 1 \\
\hline \multirow{13}{*}{$\begin{array}{l}\text { Antibiotic } \\
\text { type }\end{array}$} & Colistin $(\mathrm{N}=50)$ & $26(52 \%)$ & 0.076 \\
\hline & Imipenem $(\mathrm{N}=35)$ & $12(34.3 \%)$ & 0.249 \\
\hline & Meropenem $(\mathrm{N}=29)$ & $14(48.2 \%)$ & 0.465 \\
\hline & Ciprofloxacin( $\mathrm{N}=8)$ & $4(50 \%)$ & 0.721 \\
\hline & Levofloxacin $(\mathrm{N}=4)$ & $3(75 \%)$ & 0.310 \\
\hline & Amikacin $(\mathrm{N}=36)$ & $8(22.2 \%)$ & 0.004 \\
\hline & $\operatorname{Gentamicin}(\mathrm{N}=5)$ & $0(0 \%)$ & 0.072 \\
\hline & Cefotaxime $(\mathrm{N}=2)$ & $2(100 \%)$ & 0.178 \\
\hline & Ceftriaxone $(\mathrm{N}=2)$ & $1(50 \%)$ & 1 \\
\hline & Ceftazidime $(\mathrm{N}=3)$ & $1(33.3 \%)$ & 1 \\
\hline & Cefepime(N=6) & $4(66.6 \%)$ & 0.400 \\
\hline & $\begin{array}{l}\text { Piperacillin- } \\
\text { Tazobactam }(\mathrm{N}=10)\end{array}$ & $9(90 \%)$ & 0.001 \\
\hline & $\begin{array}{l}\text { Trimethoprime- } \\
\text { Sulfamethoxazole }(\mathrm{N}=2)\end{array}$ & $0(0 \%)$ & 0.508 \\
\hline
\end{tabular}

With regard to Fluoroquinolones, CRE were highly resistant to these antibiotics. The highest resistance rates were to Norfloxacin $95.1 \%$, followed by Ciprofloxacin $88.8 \%$, and then Levofloxacin $79.8 \%$. These were clearly 
higher than the resistance rates in other studies such as the Montagnani study [18], the Logan 2015 study [19], the Logan 2012 study [6], and the Chiotos study [20]. Nonetheless, the resistance rates in the Han's study were close to ours; they were $78.4 \%$ and $76.3 \%$ to Ciprofloxacin and Levofloxacin, respectively [1].

Concerning Carbapenems, there were high resistance rates to Doripenem and Ertapenem (96\% and 92.8\%, respectively) despite the fact that they are not used in our
[18], the Logan 2015 study (53.9\%) [19], the Logan 2012 study (68\%) [6], and the Han study (54.8\%) [1].

The resistance rate to Nitrofurantoin was $52.2 \%$ in our study. This is lower than the resistance rate in the Han study, which was $64.1 \%$ [1].

In our study, there was resistance to Colistin by $26.6 \%$. This is higher than the rates in the Montagnani study, which were $13.4 \%$ [18]. The resistance to Polymyxin in the Logan 2012 study was $4.5 \%$ [6]. The

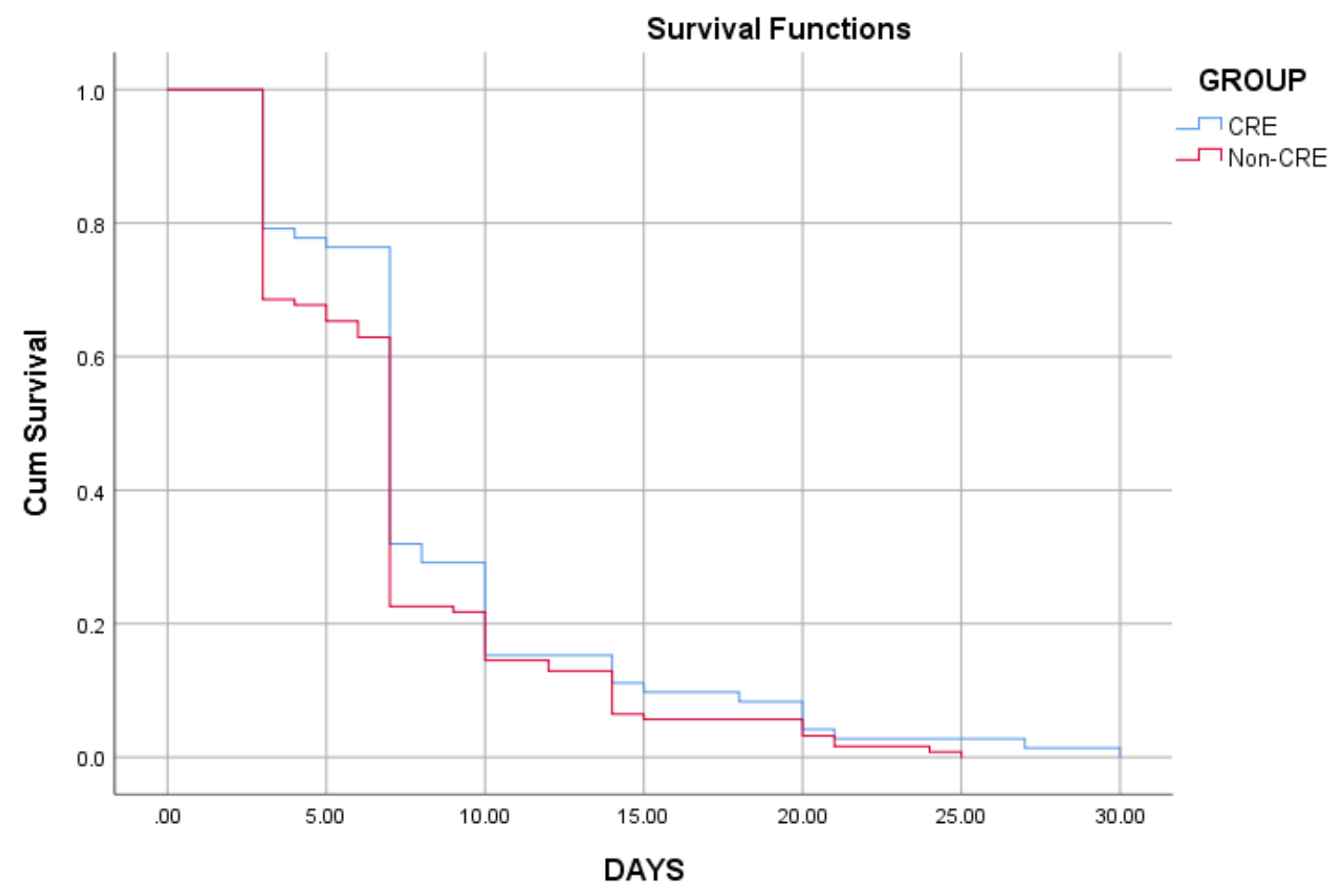

Figure 1 Survival curve; comparation between CRE \& non-CRE group according to response time.

hospital. On the other hand, the resistance rates to Imipenem and to Meropenem were $46.45 \%$ and $52.8 \%$, respectively. This is consistent with the Chiotos study; the resistance rates to Ertapenem, Imipenem and Meropenem were $95 \%, 41 \%$, and $40 \%$, respectively [20]. We also agree with the resistance rates to Ertapenem in the Montagnani study, which were $87.7 \%$. However, the resistance rates to Meropenem and Imipenem, $71.2 \%$ and $69.4 \%$, respectively, were higher in the Montagnani study [18]. The Logan 2015 study was similar to our study regarding the resistance rate to Meropenem 54\%, while it was higher to Imipenem $82 \%$ and lower to Ertapenem 59\% [19]. Also, the Han study had high levels of resistance to Imipenem, Meropenem and Ertapenem (96.1\%, 97\%, 98.9\%, respectively) [1]. No sensitivity to Doripenem was carried out in these studies.

In our study, there was a high resistance rate to Sulfamethoxazole with Trimethoprim (92\%), which is higher than the ratios in the Montagnani study (69.7\%) rate in the Chiotos study was $20 \%$ [20], and in the Han study was $4 \%$ [1]. There was no resistance to Colistin in the Ding study in China [21].

There was no resistance to Fosfomycin in the Ding study [21], while in our study, the resistance rate to Fosfomycin was 59.3\%. Also, the Chiotos 2016 study had indicated an efficacy of Fosfomycin in treating CRE in children [13].

In our study, the resistance rate to Tigecycline was $13.3 \%$ with a high intermediate resistance rate of $40 \%$. This is less than the ratio in the Montagnani study $(22.7 \%)$ [18], and close to the rate in the Chiotos study (46\%). The Chiotos study relied on (EUCAST) to determine the resistance where the minimum inhibitory concentration was more than $2 \mathrm{mcg} / \mathrm{ml}$ [20],this is equal to the intermediate resistance in our study. In comparison to the Logan and Han studies, the resistance rate in our study was clearly higher [1] [6]. 
When comparing these resistance ratios with the NonCRE group, we notice that the resistance rates were lower towards most of the other antibiotic groups. The resistance rates to Aminoglycosides were the following: Amikacin and Gentamicin were $7.2 \%$ and $47.4 \%$, respectively. Nevertheless, the resistance rate to Cephalosporins was high, especially to Ceftriaxone and Cefotaxime, and was somewhat low to Cefepime and Ceftazidime. In addition, high resistance to Amoxicillin with Clavulanates and lower levels of resistance rates to Piperacillin with Tazobactam were recorded. Concerning Fluoroquinolones, the resistance rates were lower $(20.4 \%$ $-40.85 \%)$. The resistance rate to Trimethoprim with Sulfamethoxazole was also lower. The high intermediate resistance rate for Nitrofurantoin was $48.3 \%$, and the resistance to Colistin was low $(7.2 \%)$.

The mortality rate was very high $42.4 \%$ (30.4\% of which was mainly due to infection). This rate is higher than the ones mentioned in the Chiotos study (14\%) [22], and in the Logan 2012 study (10\%) [6]. No deaths were recorded in the CRE group in the Alzomor study [17]. This rate in our study was statistically significant compared to the Non-CRE group, which had a mortality rate of $18.4 \%$, and deaths due to infection itself accounted for $12.5 \%$.

The most commonly used antibiotics in our study were Colistin, Imipenem, and Amikacin. They were used as a single treatment or in combination with other antibiotics. The use of Colistin is associated with a mortality rate higher than other antibiotics; however, it wasn't as statically significant as its role in the mortality rate in other studies like the Montagnani study [18]. In comparison, the significance of Piperacillin-Tazobactam in increasing the mortality rate was reported in our study when used in the treatment plan. The treatments that used Amikacin alone or in combination with other antibiotics had a statistically significant lower mortality rate.

Regarding gender, there was no statistical significance in the mortality rate as in the Montagnani study [18].

We found a statistically significant higher risk of mortality in neonate age, whereas the higher mortality risk was in ages more than 24 months in the Montagnani study [18]. The bacterial species was not important in increasing the mortality rate in our study, which is consistent with the Montagnani study [18].

We found that urine samples reflecting infection within the urinary tract were associated with a statistically significant low mortality rate in the CRE group. The bronchial lavage samples reflecting respiratory infections were associated with a statistically significant increase in mortality, and this was also reported in the Montagnani study [18].

The largest proportion of patients received a single treatment. The median duration of response to treatment was 8.4 (5.5) days. In comparison to the Non-CRE group, there was no statistically significant difference in terms of the time required for response. We also found no significance to the number of antibiotics in the mortality rate, while there was a statistically significant difference in cases in which antibiotics were not used; death occurred in all cases. However, in the Montagnani study, the use of three or more antibiotics was associated with a statistically significant increase in the mortality rate [18].

\section{CONCLUSION}

In conclusion, our study focuses on CRE in children. It is the first study in Syria that provides a valuable epidemiology and clinical data, and determines the benefit of our treatment as we have noticed. Our treatment options were limited; therefore, Colistin is the empiric antibiotic choice. This requires the introduction of novel antibiotics in our treatment options.

The Limitation of our study was that no molecular study was performed to investigate the presence of Carbapenemase production.

\section{REFERENCES}

[1] R. Han et al., "Dissemination of Carbapenemases (KPC, NDM, OXA-48, IMP, and VIM) Among Carbapenem-Resistant Enterobacteriaceae Isolated From Adult and Children Patients in China," Front. Cell. Infect. Microbiol., vol. 10, p. 314, Jul. 2020, doi: 10.3389/fcimb.2020.00314

[2] E. Castagnolaa et al., "Epidemiology of carbapenemase-producing Enterobacteriaceae in apediatric hospital in a country with high endemicity," J. Infect. Public Health, vol. 12, pp. 270-274, 2019, doi: 10.1016/j.jiph.2018.11.003.

[3] Center for Disease Control and Prevention (CDC), "FAQs about choosing and implementing a CRE definition.," Jun. 2015, [Online]. Available: http://www

cdc.gov/hai/organisms/cre/definition.html.

[4] H. Z. Smith and B. Kendall, "Carbapenem Resistant Enterobacteriacea (CRE)," StatPearls [Internet]. Treasure Island (FL): StatPearls Publishing; 2020 Jan-., 2020.

[5] J. C. Cho, M. T. Zmarlicka, K. M. Shaeer, and J. Pardo, "Meropenem/Vaborbactam, the First Carbapenem/ $\beta$-Lactamase Inhibitor Combination," Ann. Pharmacother., vol. 52, no. 8, pp. 769-779, 2018, doi: https://doi.org/10.1177/10600280187632.

[6] L. K. Logan, "Carbapenem-Resistant Enterobacteriaceae: An Emerging Problem in Children," Clin. Infect. Dis., vol. 55, no. 6, pp. 852 859, 2012, doi: 10.1093/cid/cis543. 
[7] K. Chiotos, M. Hayes, J. S. Gerber, and P. D. Tamma, "Treatment of Carbapenem-Resistant Enterobacteriaceae Infections in Children," J. Pediatr. Infect. Dis. Soc., vol. 9, no. 1, pp. 56-66, 2020, doi: 10.1093/jpids/piz085.

[8] C. Antachopoulos and E. Iosifidis, "Colistin Use in Neonates and Children With Infections Due to Carbapenem-resistant Bacteria," Pediatr. Infect. Dis. J., vol. 36, no. 9, pp. 905-907, Sep. 2017, doi: 10.1097/INF.0000000000001655.

[9] F. Perez, N. G. El Chakhtoura, K. M. Papp-Wallace, B. M. Wilson, and R. A. Bonomo, "Treatment options for infections caused by carbapenemresistant Enterobacteriaceae: can we apply 'precision medicine' to antimicrobial chemotherapy?," Expert Opin. Pharmacother., vol. 17, no. 6, pp. 761-781, 2016, doi: 10.1517/14656566.2016.1145658.

[10] M. Vincenza Mastrolia, L. Galli, M. De Martino, and E. Chiappini, "Use of tigecycline in pediatric clinical practice," Expert Rev. Anti Infect. Ther., vol. 15, no. 6, pp. 605-612, 2017, doi: 10.1080/14787210.2017.1318064.

[11] C.-C. Sheu, Y.-T. Chang, S.-Y. Lin, Y.-H. Chen, and P.-R. Hsueh, "Infections Caused by Carbapenem-Resistant Enterobacteriaceae: An Update on Therapeutic Options," Front. Microbiol., vol. 10, no. 80, 2019, doi: 10.3389/fmicb.2019.00080.

[12] F. Baquero-Artigao and T. del Rosal Rabes, "Fosfomycin in the pediatric setting: Evidence and potential indications,” Soc. Esp. Quimioter., vol. 32, no. 1, pp. 55-61, 2019.

[13] K. Chiotos, J. H. Han, and P. D. Tamma, "Carbapenem-Resistant

Enterobacteriaceae Infections in Children," Curr Infect Dis Rep., vol. 18, no. 1, Jan. 2016, doi: 10.1007/s11908-015-05109.

[14] Becton, Dickinson and Company, BD BBL SensiDisc Antimicrobial Susceptibility Test Discs. .

[15] "Epidemiology and burden of disease of carbapenem-resistant Enterobacteriaceae (CRE),Acinetobacter baumannii (CRAB) and Pseudomonas aeruginosa (CRPsA)," in Guidelines for the prevention and control of carbapenemresistant Enterobacteriaceae, Acinetobacter baumannii and Pseudomonas aeruginosa in health care facilities., Geneva, Switzerland: WHO Document Production Services, 2017, pp. 19-21.

[16] P. K. Nair and M. S. Vaz, "Prevalence of carbapenem resistant Enterobacteriaceae from a tertiary care hospital in Mumbai, India," J. Microbiol. Infect. Dis., vol. 3, no. 4, pp. 207-210, 2013, doi: 10.5799/ahinjs.02.2013.04.0110.

[17] O. A. Alzomor, T. S. Alfawaz, A. Abu-Shaheen, M. A. Alshehri, and D. Al Shahrani, "A matched casecontrol study to assess the carbapenemresistant Enterobacteriaceae infections among hospitalized children at King Fahad Medical City, Riyadh, Saudi Arabia," Saudi Med J, vol. 40, no. 11, pp. 179-184, 2019, doi: 10.15537/smj.2019.11.24586.

[18] C. Montagnani et al., "Carbapenem-Resistant Enterobacteriaceae Infections in Children: An Italian Retrospective Multicenter Study," Pediatr. Infect. Dis. J., vol. 35, no. 8, pp. 862-868, 2016, doi: 10.1097/INF.0000000000001188.

[19] L. K. Logan, J. P. Renschler, S. Gandra, R. A. Weinstein, and R. Laxminarayan, "CarbapenemResistant Enterobacteriaceae in Children, United States, 1999-2012," Emerg. Infect. Dis., vol. 21, no. 11, pp. 2014-2021, Nov. 2015, doi: 10.3201/eid2111.150548.

[20] K. Chiotos et al., "Multicenter Study of the Risk Factors for Colonization or Infection with Carbapenem-Resistant Enterobacteriaceae in Children," Antimicrob. Agents Chemother., vol. 61, no. 12, pp. e01440-17, Dec. 2017, doi: https://doi.org/10.1128/AAC .01440-17.

[21] Y. Ding, Y. Wang, Y. Hsia, M. Sharland, and P. T. Heath, "Systematic review of carbapenem-resistant Enterobacteriaceae causing neonatal sepsis in China," Ann. Clin. Microbiol. Antimicrob., vol. 18, no. 1, pp. 1-8, 2019, doi: 10.1186/s12941-0190334-9.

[22] K. Chiotos et al., "CARBAPENEM-RESISTANT ENTEROBACTERIACEAE: AN EMERGING PEDIATRIC PATHOGEN," Crit. Care Med., vol. 44, no. 12, p. 238, 2016, doi: 10.1097\01.ccm.0000509324.10405.40. 